\title{
Review Article \\ Surveillance, Phagocytosis, and Inflammation: How Never-Resting Microglia Influence Adult Hippocampal Neurogenesis
}

\author{
Amanda Sierra, ${ }^{1,2,3}$ Sol Beccari, ${ }^{2,3}$ Irune Diaz-Aparicio, ${ }^{2,3}$ Juan M. Encinas, ${ }^{1,2,3}$ \\ Samuel Comeau, ${ }^{4,5}$ and Marie-Ève Tremblay ${ }^{4,5}$ \\ ${ }^{1}$ Ikerbasque Foundation, 48011 Bilbao, Spain \\ ${ }^{2}$ Achucarro Basque Center for Neuroscience, Bizkaia Science and Technology Park, 48170 Zamudio, Spain \\ ${ }^{3}$ Department of Neurosciences, University of the Basque Country, 48940 Leioa, Spain \\ ${ }^{4}$ Centre de Recherche du CHU de Québec, Axe Neurosciences, Canada G1P 4C7 \\ ${ }^{5}$ Département de Médecine Moléculaire, Université Laval, Canada G1V 4 G2
}

Correspondence should be addressed to Amanda Sierra; a.sierra@ikerbasque.org and Marie-Ève Tremblay; Tremblay.Marie-Eve@crchudequebec.ulaval.ca

Received 10 December 2013; Accepted 11 February 2014; Published 19 March 2014

Academic Editor: Carlos Fitzsimons

Copyright (C) 2014 Amanda Sierra et al. This is an open access article distributed under the Creative Commons Attribution License, which permits unrestricted use, distribution, and reproduction in any medium, provided the original work is properly cited.

Microglia cells are the major orchestrator of the brain inflammatory response. As such, they are traditionally studied in various contexts of trauma, injury, and disease, where they are well-known for regulating a wide range of physiological processes by their release of proinflammatory cytokines, reactive oxygen species, and trophic factors, among other crucial mediators. In the last few years, however, this classical view of microglia was challenged by a series of discoveries showing their active and positive contribution to normal brain functions. In light of these discoveries, surveillant microglia are now emerging as an important effector of cellular plasticity in the healthy brain, alongside astrocytes and other types of inflammatory cells. Here, we will review the roles of microglia in adult hippocampal neurogenesis and their regulation by inflammation during chronic stress, aging, and neurodegenerative diseases, with a particular emphasis on their underlying molecular mechanisms and their functional consequences for learning and memory.

\section{Microglia: The Resident Immune Cells of the Brain}

Microglia were first described in 1919 by the Spanish neuroanatomist Pío del Río Hortega, a disciple of the renowned Santiago Ramón y Cajal, almost half a century later than neurons and astrocytes and just before oligodendrocytes [1]. This delayed appearance into the neuroscience arena is still apparent today, as microglia remain one of the least understood cell types of the brain. Traditionally, microglia were simply considered as "brain macrophages" controlling the inflammatory response during acute insults and neurodegenerative conditions, and only recently was their unique origin revealed. Indeed, microglia were shown to derive from primitive myeloid progenitors of the yolk sac that invade the central nervous system (CNS) during early embryonic development (reviewed in [2]). In contrast, circulating monocytes and lymphocytes, as well as most tissue macrophages, derive from hematopoietic stem cells located initially in the foetal liver and later in the bone marrow [3]. In the adult brain, the microglial population is maintained exclusively by self-renewal during normal physiological conditions [2]. As a consequence, microglia are the only immune cells which permanently reside in the CNS parenchyma, alongside neural tube-derived neurons, astrocytes, and oligodendrocytes.

These past few years, unprecedented insights were also provided into their extreme dynamism and functional behaviour, in health as much as in disease. Indeed, microglia 
were revealed to be exceptional sensors of their environment, responding on a time scale of minutes to even subtle variations of their milieu, by undergoing concerted changes in morphology and gene expression $[4,5]$. During pathological insults, "activated" microglia were particularly shown to thicken and retract their processes, extend filopodia, proliferate and migrate, release factors and compounds influencing neuronal survival (such as proinflammatory cytokines, trophic factors, reactive oxygen species (ROS), etc.), and phagocytose pathogens, degenerating cells and debris, thus providing better understanding of their roles in orchestrating the inflammatory response [6]. These abilities as immune cells are also recruited during normal physiological conditions, where "surveillant" microglia further participate in the remodeling of neuronal circuits by their phagocytic elimination of synapses and their regulation of glutamatergic receptors maturation and synaptic transmission, among other previously unexpected roles [7-9], in addition to their crucial involvement in the phagocytic elimination of newborn cells in the context of adult neurogenesis [10].

Our review will discuss the emerging roles of microglia in adult hippocampal neurogenesis and their regulation by inflammation during chronic stress, aging, and neurodegenerative diseases, with a particular emphasis on their underlying molecular mechanisms and their functional consequences for learning and memory (Figure 1).

\section{A Brief Overview of Adult Hippocampal Neurogenesis}

Adult hippocampal neurogenesis is continuously maintained by the proliferation of neural stem cells located in the subgranular zone (SGZ) [11-13]. These neuroprogenitors have been named "radial glia-like cells" (rNSCs), or type 1 cells, since they morphologically and functionally resemble the embryonic radial glia. They have also been defined as "quiescent neuroprogenitors" because only a small percentage of the population is actively dividing during normal physiological conditions. The lineage of these cells is frequently traced by using analogs of the nucleotide thymidine, such as bromodeoxyuridine (BrdU) which gets incorporated into the DNA of dividing cells during the $S$ phase and can be detected by immunofluorescence. Alternatively, their lineage can be traced by labeling with fluorescent reporters which are delivered to dividing cells by retroviral vectors or expressed by specific cell type promoters via inducible transgenic mice (for a review of the methods commonly used to study adult neurogenesis, see [14]). The daughter cells of rNSCs, also called type 2 cells or amplifying neuroprogenitors (ANPs), rapidly expand their pool by proliferating before becoming postmitotic neuroblasts. Within a month, these neuroblasts differentiate and integrate as mature neurons into the hippocampal circuitry [15]. They however display unique electrophysiological characteristics during several months, being more excitable than mature neurons [16], and constitute a special cell population that is particularly inclined to undergo synaptic remodeling and activity-dependent plasticity [17].
These unique properties of the newborn neurons and the neurogenic cascade in general suggested that adult hippocampal neurogenesis could play an important role in hippocampal-dependent functions that require extensive neuroplasticity such as learning and memory. Indeed, activity-dependent plasticity and learning are long known for modulating adult neurogenesis in a complex, yet specific manner, with adult hippocampal neurogenesis being influenced by learning tasks which depend on the hippocampus $[44,45]$. For instance, hippocampal-dependent learning paradigms were found to regulate the survival of newborn neurons, in a positive manner that depends on the timing between their birth and the phases of learning $[46,47]$. Young (1.5-2 months old) newborn neurons were also shown to be preferentially activated during memory recall in a water maze task, compared to mature neurons, as determined by colabeling of BrdU with immediate early genes such as cFos and Arc, in which expression correlates with neuronal firing [48]. Nonetheless, it has only been in the last few years that loss-of-function and gain-of-function approaches with inducible transgenic mice were able to confirm that adult hippocampal neurogenesis is necessary for synaptic transmission and plasticity, including the induction of longterm potentiation (LTP) and long-term depression [49], as well as trace learning in conditioned protocols [50], memory retention in spatial learning tasks [51,52], and encoding of overlapping input patterns, that is, pattern separation [53].

Adult hippocampal neurogenesis and its functional implications for learning and memory are however influenced negatively by a variety of conditions that are commonly associated with microglial activation and inflammation in the brain, such as chronic stress, aging, and neurodegenerative diseases, as we will review herein. Indeed, inflammation caused by irradiation produces a sustained inhibition of neurogenesis, notably by decreasing the proliferation and neuronal differentiation of the progenitors, and therefore, exposure to therapeutic doses of cranial irradiation has been widely used for modulating neurogenesis experimentally before the development of more specific approaches [54].

\section{Regulation of Adult Hippocampal Neurogenesis by Inflammation}

Inflammation is a natural bodily response to damage or infection that is generally mediated by proinflammatory cytokines such as interleukin 1 beta (IL-1 $\beta$ ), interleukin 6 (IL6 ), and tumour necrosis factor alpha (TNF $\alpha$ ), in addition to lipidic mediators such as prostaglandins and leukotrienes. Oftentimes, it is associated with an increased production of ROS, as well as nitric oxide (NO). Together, these proinflammatory mediators lead to an increase in local blood flow, adhesion, and extravasation of circulating monocytes, neutrophils, and lymphocytes [55]. In the brain, microglia are the main orchestrator of the neuroinflammatory response, but other resident cell types, including astrocytes, endothelial cells, mast cells, perivascular and meningeal macrophages, and even neurons, can produce proinflammatory mediators, though perhaps not to the same extent as microglia [56]. 


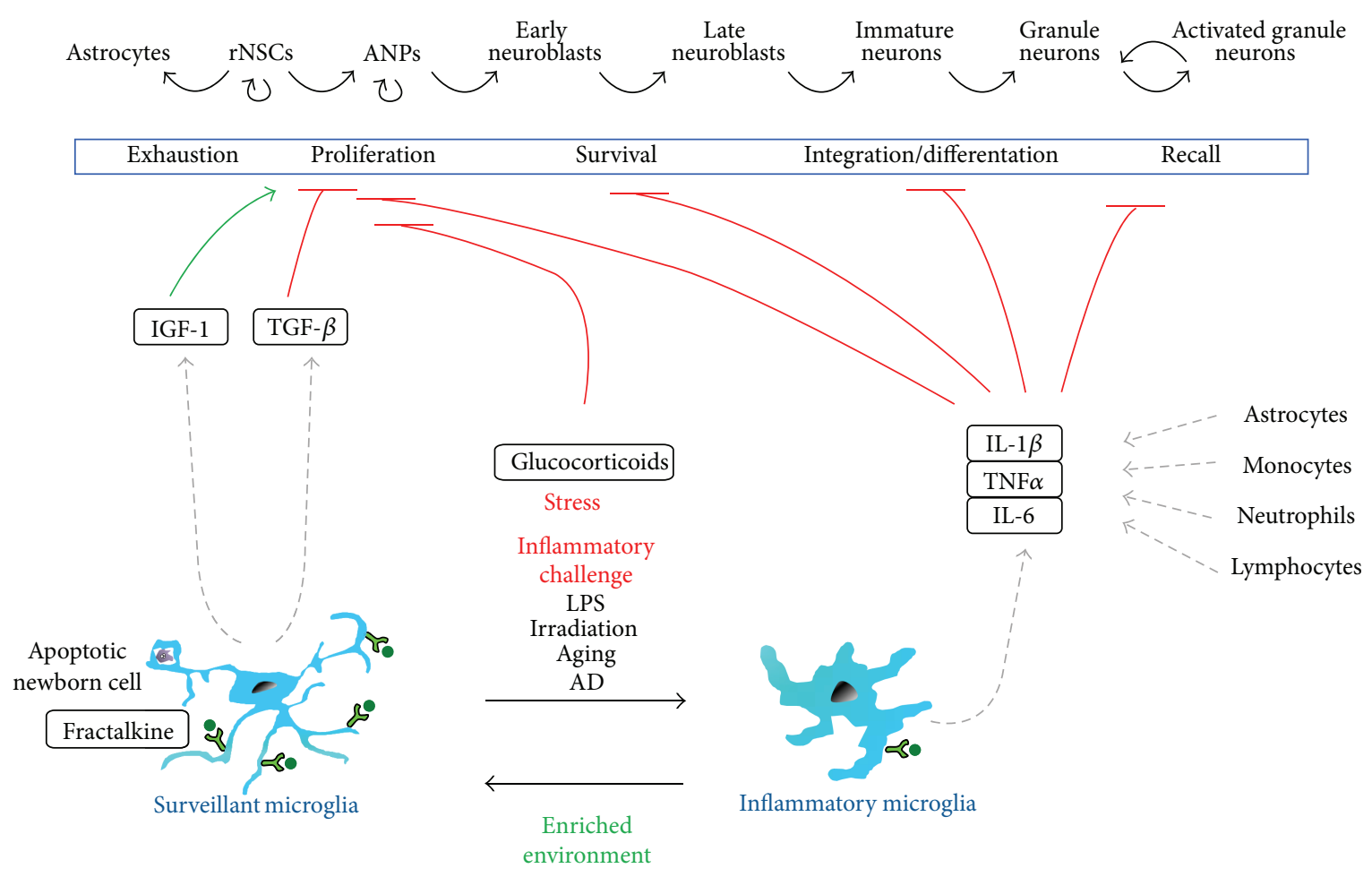

FIGURE 1: The effects of surveillant and inflammatory microglia on the adult hippocampal neurogenic cascade. During physiological conditions, surveillant microglia effectively phagocytose the excess of apoptotic newborn cells and may release antineurogenic factors such as TGF $\beta$. This anti-inflammatory state is maintained by neuronal (tethered or released) fractalkine. Enriched environment drives microglia towards a phenotype supportive of neurogenesis, via the production of IGF-1. In contrast, inflammatory challenge triggered by LPS, irradiation, aging, or AD induces the production of proinflammatory cytokines such as IL-1 $\beta$, TNF $\alpha$, and IL- 6 by microglia as well as resident astrocytes and infiltrating monocytes, neutrophils, and lymphocytes. These cytokines have profound detrimental effects on adult neurogenesis by reducing the proliferation, survival, integration, and differentiation of the newborn neurons and decreasing their recall during learning and memory paradigms.

In addition, peripheral immune cells invading the CNS during inflammation can further produce proinflammatory mediators, but the respective contribution of microglia versus other cell types in the inflammatory response of the brain is poorly understood.

The harmful effects of inflammation are also widely determined by the actual levels of proinflammatory mediators released, rather than the occurrence or absence of an inflammatory response in itself. For instance, $\mathrm{TNF} \alpha$ regulates synaptic plasticity by potentiating the cell surface expression of AMPA glutamatergic receptors, thus resulting in a homeostatic scaling following prolonged blockage of neuronal activity during visual system development [57]. However, TNF $\alpha$ also produces differential effects at higher concentrations, ranging from an inhibition of long-term potentiation to an enhancement of glutamate-mediated excitotoxicity in vitro [58]. Inflammation induced by chronic ventricular infusion of bacterial lipopolysaccharides (LPS; a main component of the outer membrane of Gram-negative bacteria), that is, the most widely used method for inducing an inflammatory challenge, also increases ex vivo the hippocampal levels of TNF $\alpha$ and IL-1 $\beta$, thereby impairing novel place recognition, spatial learning, and memory formation, but all these cognitive deficits can be restored by pharmacological treatment with a TNF $\alpha$ protein synthesis inhibitor, a novel analog of thalidomide, 3,6 ${ }^{\prime}$-dithiothalidomide [59].

The impact of inflammation on adult hippocampal neurogenesis was originally discovered by Olle Lindvall and Theo Palmer's groups in 2003, showing that systemic or intrahippocampal administration of LPS reduces the formation of newborn neurons in the adult hippocampus, an effect that is prevented by indomethacin, a nonsteroidal antiinflammatory drug (NSAID) which inhibits the synthesis of proinflammatory prostaglandins $[60,61]$. Similarly, inflammation can determine the increase in neurogenesis that is driven by seizures, a context in which neurogenesis can be prevented by LPS and increased by the anti-inflammatory antibiotic minocycline [60]. In these studies, hippocampal proliferation remained unaffected by LPS or minocycline and thus it is likely that inflammation targeted the survival of newborn cells $[60,61]$, as LPS is known to increase SGZ apoptosis [62]. Inflammation also has further downstream effects on the neurogenic cascade. For instance, LPS increases the number of thin dendritic spines and the expression of the excitatory synapses marker "postsynaptic density protein of 95kDa" (PSD95) in newborn neurons. LPS in addition increases the expression of $\mathrm{GABA}_{\mathrm{A}}$ receptors at early stages of synapse formation, leading to suggesting a possible 
imbalance of excitatory and inhibitory neurotransmission in these young neurons [63]. Finally, LPS also prevents the integration of newborn neurons into behaviourally relevant networks, including most notably their activation during spatial exploration, as determined by the percentage of BrdU cells colabeled with the immediate early gene Arc [64].

Importantly, none of these manipulations is specific to microglia and may directly or indirectly affect other brain cells involved in the inflammatory response of the brain. For instance, both LPS and minocycline affect astrocytic function in vitro and in vivo [65-69]. Furthermore, LPS is known to drive infiltration of monocytes and neutrophils into the brain parenchyma [70]. Monocytes and neutrophils produce major proinflammatory mediators and could therefore act on the neurogenic cascade as well. The implication of microglia in LPS-induced decrease in neurogenesis is nonetheless supported in vivo by the negative correlation between the number of newborn neurons (BrdU+, NeuN+ cells) and the number of "activated" microglia (i.e., expressing ED1) [60]. ED1, also called CD68 or macrosialin, is a lysosomal protein which is overexpressed during inflammatory challenge. While the location of ED1 previously suggested its involvement in phagocytosis, its loss of function did not result in phagocytosis deficits and thus, its function still remains unknown (reviewed in [10]). The number of ED1-positive microglia also negatively correlates with neurogenesis during inflammation provoked by cranial irradiation [61]. While correlation does not involve causation, nor can pinpoint to the underlying mechanism, these experiments were the first to reveal a potential role for "activated" microglia in the regulation of adult hippocampal neurogenesis. More direct evidence of microglial mediation in LPS deleterious effects was obtained from in vitro experiments, as it was shown that conditioned media from LPS-challenged microglia contained IL-6, which in turn caused apoptosis of neuroblasts [61]. Nonetheless, astrocytes can also release IL- 6 when stimulated with $\mathrm{TNF} \alpha$ or IL-1 $\beta$ [71] and chronic astrocytic release of IL-6 in transgenic mice reduced proliferation, survival, and differentiation of newborn cells, thus resulting in a net decrease in neurogenesis [72]. In summary, while the detrimental impact of inflammation on neurogenesis is well established, more work is needed to define the specific roles played by the various inflammatory cells populating the brain.

\section{Inflammation Associated with Chronic Stress}

Across health and disease, the most prevalent condition that is associated with neuroinflammation is "chronic stress," which commonly refers to the repeated or sustained inability to cope with stressful environmental, social, and psychological constraints. Chronic stress is characterized by an imbalanced secretion of glucocorticoids by the hypothalamic-pituitary-adrenal (HPA) axis (most notably cortisol in humans and corticosterone in rodents), which leads to an altered brain remodeling, massive loss of synapses, and compromised cognitive function [73]. In particular, an impairment of spatial learning, working memory, novelty seeking, and decision making has been associated with chronic stress [74]. Glucocorticoids are well known for their anti-inflammatory properties, as they interfere with NF- $\kappa$ B-mediated cytokine transcription, ultimately delaying wound healing [75]. They are also potent anti-inflammatory mediators in vivo [76] and in purified microglia cultures [77]. Recently, repeated administration of high doses of glucocorticoids by intraperitoneal injection, to mimic their release by chronic stress, was also shown to induce a loss of dendritic spines in the motor cortex, while impairing learning of a motor task. A transcription-dependent pathway acting downstream of the glucocorticoid receptor GR was proposed $[78,79]$ but the particular cell types involved were not identified.

Microglia are considered to be a direct target of the glucocorticoids, as they were shown to express GR during normal physiological conditions in vivo [77]. In fact, transgenic mice lacking GR in microglia and macrophages show an increased production of proinflammatory mediators (including TNF $\alpha$ and IL-1 $\beta$ ) and greater neuronal damage in response to an intraparenchymal injection of LPS, compared to wild-type mice [80]. In contrast, glucocorticoids are considered to be proinflammatory in the chronically stressed brain [81], where among other changes they can promote inflammation, oxidative stress, neurodegeneration, and microglial activation [82]. For example, repeated restraint stress induces microglial proliferation and morphological changes, including a hyperramification of their processes in the adult hippocampus following restraint stress [83], but a nearly complete loss of processes in the context of social defeat [84]. Prenatal restraint stress also causes an increase in the basal levels of TNF $\alpha$ and IL- $1 \beta$, while increasing the proportion of microglia showing a reactive morphology in the adult hippocampus [85]. Similarly, social defeat leads to an enhanced response to the inflammatory challenge induced by intraperitoneal injection of LPS, including an increased production of TNF $\alpha$ and IL- $1 \beta$, and expression of inducible NO synthase (iNOS) by microglia, accompanied by an increased infiltration of circulating monocytes $[84,86]$. Therefore, microglia are a strong candidate for mediating some of the effects of stress on adult neurogenesis, as will be discussed below, in synergy with other types of inflammatory cells.

Chronic stress is well known for its negative effects on hippocampal neurogenesis (reviewed in $[87,88]$ ), although not all stress paradigms are equally effective [89]. Several stress paradigms can decrease neuroprogenitors proliferation in the tree shrew [90] and in mice [91, 92], although this effect seems to be compensated by an increased survival of newborn neurons [92] and whether stress results in a net increase or decrease in neurogenesis remains controversial (reviewed in $[87,88])$. The effects of stress on adult neurogenesis seem to be mediated at least partially by glucocorticoids, because mice lacking a single copy of the GR gene show behavioural symptoms of depression including learned helplessness, neuroendocrine alterations of the HPA axis, and impaired neurogenesis [93]. In parallel, chronic stress is associated with an increased inflammatory response, which may inhibit neurogenesis as well. For instance, serum levels 
of IL-1 $\beta$ and IL- 6 are significantly increased in depressed patients [94]. In mice, restraint stress leads to a widespread activation of NF- $\kappa \mathrm{B}$ in the hippocampus, including at the level of neuroprogenitors [95] and increased protein levels of IL-1 $\beta$ [96]. In addition to the direct role of glucocorticoids, IL-1 $\beta$ also seems to mediate some of the effects of mild chronic stress, because in vivo manipulations that block IL-1 $\beta$ (either pharmacologically or in null transgenic mice) prevent the anhedonic stress response and the antineurogenic effect of stress $[91,96]$. Moreover, the corticoids and IL- $1 \beta$ pathways may regulate each other in a bidirectional manner because the administration of a GR antagonist can blunt the LPSinduced production of hippocampal IL- $1 \beta$ in stressed mice [97], whereas mice knockout for the IL-1 $\beta$ receptor (IL-1R1) fail to display the characteristic elevation of corticosterone induced by mild chronic stress [96]. Another stress-related cytokine, IL-6, induces depressive phenotypes and prevents the antidepressant actions of fluoxetine when administered to mice in vivo [98]. So far the effects of stress on neurogenesis via corticosteroids and inflammation have been assumed to be cell autonomous, as neuroprogenitors express both GR [99] and IL-1R1 [95]. The potential participation of microglia is yet to be determined, but there are some reports of a direct effect of stress on microglial activation. For instance, microglia acutely isolated from mice subjected to acute stress (by inescapable tail shock) showed a primed response to LPS challenge by producing higher levels of IL- $1 \beta$ mRNA ex vivo [100], and the specific loss of expression of GR in microglia leads to a blunted inflammatory response in vitro and to a decreased neuronal damage in vivo in response to LPS [80]. In stress paradigms, these enhanced responses of microglia to inflammatory challenges are similar to their age-related "priming" which has been associated with and is possibly due to an increased basal production of proinflammatory mediators. However, whether microglia express increased levels of IL-1 $\beta$ and other proinflammatory cytokines in response to stressful events is presently unclear [101]. It is thus possible that some of the antineurogenic effects of stress are exerted by means of microglial-dependent inflammation, but this hypothesis remains to be experimentally tested.

\section{Inflammation Associated with Aging and Neurodegenerative Diseases}

Inflammation is also commonly associated with normal aging and neurodegenerative diseases and, therefore, could represent a putative underlying mechanism that explains their decrease in hippocampal neurogenesis. Nonetheless, inflammation is also associated with neurological diseases, such as epilepsy or stroke, where neurogenesis is thought to be increased, although the data from rodents and humans is somewhat conflictive [102]. Neurogenesis is well known to decline throughout adulthood and normal aging in rodents and humans $[103,104]$, but the decay is more pronounced and occurs later in life in mice than in humans [105]. The agingassociated decrease in neurogenesis has been shown to occur mainly as a consequence of exhaustion of the rNSC population which, after being recruited and activated, undergo three rounds of mitosis in average and then terminally differentiate into astrocytes $[12,106]$. In addition, a reduced mitotic capacity of the neuroprogenitors could further contribute to decreasing neurogenesis [106], and moreover, an age-related increase in the levels of proinflammatory cytokines could also hinder neurogenesis in the aging brain. Serum levels of IL- $1 \beta$, IL- 6 , and TNF $\alpha$ are elevated in elderly patients $[107,108]$. Aged microglia express higher levels of these proinflammatory cytokines and show a greater response to LPS inflammatory challenge, that is, a "primed" response, than their younger counterparts [109]. The origin of this low-grade age-related inflammation ("inflamm-aging" [110]) remains unknown and may be related to both aging and damage to the surrounding neurons, as well as aging of the immune system per se.

At the cellular level, stress to the endoplasmic reticulum (ER) caused by various perturbations, such as nutrient depletion, disturbances in calcium or redox status, or increased levels of misfolded proteins, can induce a cell-autonomous inflammatory response to neurons. Stress to the ER, a multifunctional organelle which is involved in protein folding, lipid biosynthesis, and calcium storage triggers a homeostatic response mechanism named the unfolding protein response (UPR), aiming to clear the unfolded proteins in order to restore normal ER homeostasis [111]. However, if the ER stress cannot be resolved, the UPR also initiates inflammatory and apoptotic pathways via activation of the transcription factor NF- $\kappa$ B which controls the expression of most proinflammatory cytokines [112]. In the brain, ER stress is often initiated by the formation of abnormal protein aggregates in several neurodegenerative diseases such as Alzheimer's disease (AD), Parkinson's disease (PD), amyotrophic lateral sclerosis (ALS), Huntington's disease (HD), and prion-related disorders [113]. This neurodegeneration-associated ER stress is assumed to occur mostly in neurons, but there are some examples of microglial protein misfolding as well. For instance, both microglia and neurons overexpress $\mathrm{CHOP}$ (C/EBP homologous protein), a transcription factor which is activated during ER stress in human patients and mouse models of ALS [114]. Inflammation has been speculated to be a main negative contributor to the pathology of ALS [115], but a direct microglial involvement in mediating the inflammatory response to abnormal protein aggregation in ALS and other neurodegenerative conditions remains to be tested. Finally, ER stress has been linked to a variety of inflammatory conditions $[116,117]$, including chronic stress, dietinduced obesity, and drug abuse, as well as atherosclerosis and arthritis [118-120]. During normal aging, a progressive decline in expression and activity of key ER molecular chaperones and folding enzymes could also compromise the adaptive response of the UPR, thereby contributing to the age-associated decline in cellular functions [118]. Therefore, aging is strongly associated with a chronic ER stress which leads to increased activation of NF- $\kappa$ B [112]; however, the contribution of the different brain cell types to "inflammaging" is still poorly understood. The detrimental effects on neurogenesis of increased proinflammatory cytokines in the aging brain are not necessarily related to microglia, but also to stressed neurons. Furthermore, ER stress may also cause a 
cell-autonomous response in neural stem cells [121], although its impact on neurogenesis remains to be experimentally determined.

In addition, aging is accompanied by an increased level of mitochondrial oxidative stress, which in turn activates the "Inflammasome" [122], a group of multimeric proteins comprising the interleukin 1 converting enzyme (ICE, caspase 1) which serves to release the active form of the cytokine [123]. IL-1 $\beta$ may act directly on rNSCs (visualised by labeling with the Sox 2 marker), as they express IL1R1 in the adult hippocampus [91]. Treatment with IL-1 $\beta$ decreases hippocampal proliferation in young mice [91] and pharmacological inhibition of ICE partially restores the number of newborn neurons in aged mice without significantly affecting their differentiation rate [124]. Transgenic IL-1 $\beta$ overexpression results in chronic inflammation and depletion of doublecortin-labeled neuroblasts, thus mimicking the aging-associated depletion of neurogenesis [125]. The actual mechanism of action of IL- $1 \beta$ on neurogenesis in aged mice, including decreased proliferation of rNSCs/ANPs and survival of newborn neurons, remains undetermined. Microglia are a main source of IL- $1 \beta$ in the aging brain, but the hypothesis that microglia-derived IL- $1 \beta$ is responsible for depleting neurogenesis in the aging brain remains to be directly tested.

The regulation of neurogenesis by IL- $1 \beta$ in the aging brain has been further linked to the activity of another cytokine, the chemokine fractalkine, or CX3CL1. Fractalkine has soluble and membrane-tethered forms and is exclusively expressed by neurons, while the fractalkine receptor (CX3CR1) is expressed in the brain by microglia alone [126]. This module forms a unique neuron-microglia signalling unit that controls the extent of microglial inflammation in several neurodegenerative conditions including PD, ALS [127], or $\mathrm{AD}$ [128]. In fact, CX3CR1 blocking antibodies increase the production of hippocampal IL-1 $\beta$ when administered to young adult rats [129]. Importantly, chronic treatment with fractalkine increases hippocampal proliferation and the number of neuroblasts in aged (22 months old) but not young ( 3 months old) or middle-aged rats (12 months old), whereas an antagonists of CX3CR1 has the opposite effects in young, but not in middle-aged nor old rats [129]. Since fractalkine expression is decreased during aging [129], a reduced neuron-microglia signalling might be releasing the brake on microglial contribution to inflammatory responses, although increased levels of fractalkine were instead reported in aged rat hippocampus by other studies [68]. Additional insights into the role of fractalkine signalling come from knock-in mice in which the endogenous CX3CR1 locus is replaced by the fluorescent reporter GFP [126]. The initial studies suggested that $\mathrm{CX} 3 \mathrm{CR} 1^{\mathrm{GFP} / \mathrm{GFP}}$ (i.e., $\mathrm{CX} 3 \mathrm{CR} 1^{-/-}$) mice have no significant differences in brain development and functions [130], but more systematic investigations recently revealed a long list of hippocampal-dependent changes in young (3 months old) CX3CR $1^{\text {GFP/GFP }}$ and CX3CR1 $1^{\mathrm{GFP} /+}$ mice compared to wild-type mice. These changes notably included decreased neuroprogenitors proliferation and neuroblasts number, impaired LTP, performance in contextual fear conditioning and water maze spatial learning and memory, and, importantly, increased IL-1 $\beta$ protein levels [131]. The signalling pathway of fractalkine-IL-1 $\beta$ is functionally relevant, because IL-1R1 antagonists rescued LTP and cognitive function in CX3CR1 ${ }^{\text {GFP/GFP }}$ mice [131]. In sum, even though neuronal fractalkine seems to be sufficient for restraining the inflammatory activity of microglia in young rats, its downregulation during aging could activate the microglial inflammatory response and thereby subsequently reduce the proliferation of remaining neuroprogenitors.

In $\mathrm{AD}$, inflammatory cytokines such as IL- $1 \beta$ are overexpressed in the microglia associated with the amyloid beta $(\mathrm{A} \beta)$ plaques of postmortem samples [132] and in transgenic mice modeling the disease [133]. The loss of synapses (from hippocampus to frontal cortex) is one of the main pathological substrates in this disease, but adult neurogenesis is also severely reduced in most mouse models of $\mathrm{AD}$, possibly due to a decreased proliferation of neuroprogenitors and a decreased survival of newborn cells, even though the putative changes in the neurogenic cascade in postmortem samples remain controversial (reviewed in [102]). This lack of agreement is possibly explained by the fact that the vast majority of $\mathrm{AD}$ cases have a late onset over 65 years of age, when little neurogenesis remains. In contrast, in most transgenic $\mathrm{AD}$ mouse models, the $\mathrm{A} \beta$ accumulation, cognitive deficits, and changes in neurogenesis are already detectable in young animals (2-3 months old). The study of AD is further hindered by the difficulty in comparing the time course and pathology across different mouse models. For instance, early treatment with minocycline can improve cognition and reduce $\mathrm{A} \beta$ burden in mice expressing the human amyloid precursor protein (APP) [134]. In contrast, in mice expressing APP and a mutated form of presenilin 1 (PS1), which is part of the $\gamma$ secretase pathway that cleaves $A \beta$, inflammation is reduced without any detectable changes in $\mathrm{A} \beta$ plaques deposition [135]. Concomitantly with a decrease in tissue inflammatory cytokines and number of microglial cells, minocycline restores neurogenesis and hippocampus-dependent memory deficits in these APP/PS1 mice [135], indirectly suggesting that cognitive decay in $\mathrm{AD}$ may be at least in part related to a detrimental effect of inflammation on hippocampal neurogenesis. Direct evidence that neurogenesis is associated with the cognitive performance in $\mathrm{AD}$ is still lacking. Further research is also necessary to determine the neurogenic targets of $\mathrm{AD}$-related inflammation. One central open question for future therapies aiming at increasing neurogenesis and cognition in $\mathrm{AD}$ is whether neuroprogenitors are spared or whether their age-induced loss becomes accelerated. Rather than increasing the proliferation and neurogenic output of the few rNSCs remaining in an old AD brain, it may be more relevant to develop strategies that prevent the age-related loss of neuroprogenitors in presymptomatic patients.

In summary, inflammation associated with a wide variety of experimental models of disease produces strong detrimental effects on hippocampal neurogenesis. These effects on human neurogenesis are however not so well described and, in vitro, IL- $1 \beta$ increases the proliferation of hippocampal embryonic neuroprogenitors but decreases their 
differentiation into neurons [136]. Novel methods to assess hippocampal neurogenesis in the living human brain, from metabolomics of neuroprogenitors to hippocampal blood brain volume (reviewed in [102]), will help to determine the contribution of inflammation to adult neurogenesis in the healthy and diseased human brain during aging.

\section{Normal Physiological Conditions}

In the healthy mature brain, microglia are an essential component of the neurogenic SGZ niche, where they physically intermingle with neuroprogenitors, neuroblasts, and newborn neurons [62]. Here, surveillant microglia effectively and rapidly phagocytose the excess of newborn cells undergoing apoptosis [62]. Importantly, microglial phagocytosis in the adult SGZ is not disturbed by inflammation associated with aging or by LPS challenge, as the phagocytic index (i.e., the proportion of apoptotic cells completely engulfed by microglia) is maintained over $90 \%$ in these conditions [62]. Nonetheless, the consequences of microglial phagocytosis on adult hippocampal neurogenesis remain elusive. Treatment of mice with annexin $\mathrm{V}$, which binds to the phosphatidylserine (PS) receptor and prevents the recognition of PS on the surface of apoptotic cells, presumably blocking phagocytosis, increases the number of apoptotic cells in the SGZ [40]. Concomitantly, annexin $\mathrm{V}$ reduces neurogenesis by decreasing the survival of neuroblasts without affecting neuroprogenitors proliferation [40]. Similar results were obtained in transgenic mice knock-out for ELMO1, a cytoplasm protein which promotes the internalization of apoptotic cells, although the effects on neurogenesis were ascribed to a decreased phagocytic activity of neuroblasts [40]. The actual phagocytic target of the neuroblasts remains undetermined, but the newborn apoptotic cells in the adult SGZ are exclusively phagocytosed by microglia, at least in physiological conditions [62]. Nevertheless, none of the above manipulations has specifically tested the role of microglial phagocytosis in hippocampal-dependent learning and memory and thus, the functional impact of microglial phagocytosis in adult neurogenic niches during normal physiological conditions remains to be elucidated.

Microglial phagocytosis of apoptotic cells is actively anti-inflammatory, at least in vitro, and thus it has been hypothesized that anti-inflammatory cytokines produced by phagocytic microglia may further regulate neurogenesis [10]. For instance, transforming growth factor beta (TGF $\beta$ ), which is produced by phagocytic microglia in vitro [137], inhibits the proliferation of SGZ neuroprogenitors [138]. Microglia are further able to produce proneurogenic factors in vitro [139]. When primed with cytokines associated with $\mathrm{T}$ helper cells such as interleukin 4 (IL-4) or low doses of interferon gamma (IFN $\gamma)$, cultured microglia support neurogenesis and oligodendrogenesis through decreased production of TNF $\alpha$ and increased production of insulin-like growth factor 1 (IGF-1) [139], an inducer of neuroprogenitor proliferation [26]. A list of potential factors produced by microglia and known to act on neuroprogenitor proliferation can be found in Table 1. In addition, recent observations suggest that neuroprogenitor cells may not only regulate their own environment, but also influence microglial functions. For instance, vascular endothelial growth factor (VEGF) produced by cultured neuroprecursor cells directly affects microglial proliferation, migration, and phagocytosis [20]. More potential factors produced by neuroprogenitors shown to be influencing microglial activity and function can be found in Table 2. However, it has to be taken into account that most of these observations were obtained in culture and that further research is needed in order to elucidate whether those factors are also secreted and have the same regulatory responses in vivo.

In addition, microglial capacity to remodel and eliminate synaptic structures during normal physiological conditions has suggested that microglia could also control the synaptic integration of the newborn neurons generated during adult hippocampal neurogenesis [140]. Three main mechanisms were proposed: (1) the phagocytic elimination of nonapoptotic axon terminals and dendritic spines, (2) the proteolytic remodeling of the perisynaptic environment, and (3) the concomitant structural remodeling of dendritic spines $[7,140]$. Indeed, microglial contacts with synaptic elements are frequently observed in the cortex during normal physiological conditions, sometimes accompanied by their engulfment and phagocytic elimination [141-143], as in the developing retinogeniculate system [144]. Microglial cells are distinctively surrounded by pockets of extracellular space, contrarily to all the other cellular elements [142], suggesting that microglia could remodel the volume and geometry of the extracellular space, and thus the concentration of various ions, neurotransmitters, and signalling molecules in the synaptic environment. Whether microglia create the pockets of extracellular space themselves or not remains unknown, but these pockets could result from microglial release of extracellular proteases such as metalloproteinases and cathepsins [145], which are well known for influencing the formation, structural remodeling, and elimination of dendritic spines in situ and also experience-dependent plasticity in vivo $[7,146]$. More recently, microglial phagocytosis of synaptic components was also observed in the developing hippocampus, in the unique time window of synaptogenesis, a process which is notably regulated by fractalkine-CX3CR1 signalling [147]. Therefore, the attractive hypothesis that microglial sculpts the circuitry of newborn cells in the adult hippocampus deserves further attention.

Lastly, microglia were also involved in increasing adult hippocampal neurogenesis in the enriched environment (EE) experimental paradigm. EE is a paradigm mimicking some features of the normal living circumstances of wild animals, as it gives them access to social interactions, toys, running wheels, and edible treats. EE has long been known to enhance neurogenesis by acting on newborn cells survival, resulting ultimately in an enlargement of the dentate gyrus [148]. Functionally, these changes are accompanied by enhanced spatial learning and memory formation with the water maze paradigm [149]. Similar increases in neurogenesis are obtained by subjecting mice to voluntary running paradigms, although in this case the 
TABLE 1: Summary of factors secreted by microglia and the potential effect they have on neuroprogenitors in vitro.

\begin{tabular}{lcll}
\hline Microglia secreted factors & Reference & Modulation of neural progenitor cells & Reference \\
\hline BDNF & {$[18]$} & Differentiation & Survival, expansion, proliferation, differentiation \\
EGF & {$[20]$} & Survival and expansion & {$[21]$} \\
FGF $\beta$ & {$[22]$} & Survival, migration, and differentiation & [23] \\
GDNF & {$[24]$} & Proliferation & {$[25]$} \\
IGF-1 & {$[21]$} & Reduction in migration & {$[27]$} \\
IL-1 $\beta$ & {$[27]$} & Inhibition of neurogenesis \\
IL-6 & {$[28]$} & Differentiation \\
IL-7 & {$[20]$} & Differentiation \\
IL-11 & {$[20]$} & Differentiation \\
NT-4 & {$[24]$} & Expansion and differentiation \\
PDGF & {$[32]$} & Inhibition of proliferation \\
TGF $\beta$ & {$[34]$} & & {$[30]$} \\
\hline
\end{tabular}

TABLE 2: Summary of factors secreted by neuroprogenitors and the potential effect they have on microglia in vitro.

\begin{tabular}{lcll}
\hline NPC secreted factors & Reference & Modulation of microglia & Reference \\
\hline BDNF & {$[18]$} & Proliferation and induction of phagocytic activity & [35] \\
Haptoglobin & {$[24]$} & Neuroprotection & Intracellular Ca ${ }^{+2}$ elevation and proliferation \\
IL-1 $\beta$ & {$[37]$} & Increase in proliferation & {$[36]$} \\
IL-6 & {$[37]$} & Mitogen & {$[38]$} \\
M-CSF & {$[20]$} & Decrease in LPS-induced NO \\
NGF & {$[40]$} & Inhibition of TNF $\alpha$ secretion \\
TGF $\beta$ & {$[37]$} & Upregulation of IL-10 secretion \\
TNF $\alpha$ & {$[37]$} & Induction of chemotaxis and proliferation \\
VEGF & {$[20]$} & & {$[41]$} \\
\hline
\end{tabular}

effect is mediated by increased neuroprogenitor proliferation [150]. During inflammatory conditions, EE is antiapoptotic and neuroprotective [151] and it limits the hippocampal response to LPS challenge by decreasing the expression of several cytokines and chemokines, including IL1- $\beta$ and TNF $\alpha$ [152]. In fact, EE is believed to counteract the inflammatory environment and rescue the decreased number of neuroblasts in CX3CR $1^{\mathrm{GFP} / \mathrm{GFP}}$ mice compared to wild-type mice [153]. The effects of EE are independent of the IL- $1 \beta$ signalling pathway, as it increases neurogenesis in mice that are null for IL-1R1 [154]. EE also induces microglial proliferation and expression of the proneurogenic IGF-1 [155], but the full phenotype of microglia in EE compared to standard housing and its impact on the neurogenic cascade remains to be determined.

The mechanisms behind the anti-inflammatory actions of EE are unknown, but they were suggested to involve microglial interactions with $\mathrm{T}$ lymphocytes through an increased expression of the major histocompatibility complex of class II (MHC-II) during EE [155]. MHC-II is responsible for presenting the phagocytosed and degraded antigens to the antibodies expressed on the surface of a subtype of $\mathrm{T}$ lymphocytes ( $\mathrm{T}$ helper or CD4+ cells), thus initiating their activation and production of antigen-specific antibodies. Severe combined immunodeficient (SCID) mice lacking either $\mathrm{T}$ and $\mathrm{B}$ lymphocytes or nude mice lacking only $\mathrm{T}$ cells have impaired proliferation and neurogenesis in normal and EE housing compared to wild-type mice [155], as well as impaired performance in the water maze [156]. Similarly, antibody-based depletion of T helper lymphocytes impairs basal and exercise-induced proliferation and neurogenesis [157]. Furthermore, a genetic study in heterogeneous stock mice, which descend from eight inbred progenitor strains, has found a significant positive correlation between genetic loci associated to hippocampal proliferation and to the proportion of CD4+ cells among blood CD3+ lymphocytes [158]. Additional experiments are needed to fully determine the possible interactions between microglia and $\mathrm{T}$ cells in neurogenesis, because, at least in normal physiological conditions, (1) T cell surveillance of the brain parenchyma is minimal, (2) microglia are poor antigen presenting cells, and (3) antigen presentation by means of MHC-II family of molecules is thought to occur outside the brain, that is, in the meninges and choroid plexus [159]. In fact, during voluntary exercise, there are no significant changes in T cell surveillance of the hippocampus, nor a direct interaction between $\mathrm{T}$ cells and microglia, nor any changes in the gene expression profile of microglia, including that of IGF-1, IL-1 $\beta$, and TNF $\alpha$ [160]. The number of microglia is also inversely correlated with the number of hippocampal proliferating cells, rNSCs, and neuroblasts in aged ( 8 months) mice subjected to voluntary running, as well as in vitro cocultures of microglia and neuroprogenitors, which has been interpreted as resulting from an overall inhibitory effect of microglia on adult 
neurogenesis [161]. Even though EE is clearly a more complex environmental factor than voluntary running, further research is necessary to disregard nonspecific or indirect effects of genetic or antibody-based $\mathrm{T}$ cells depletion on microglia and other brain cell populations, including rNSCs. For instance, adoptive transfer of $\mathrm{T}$ helper cells treated with glatiramer acetate, a synthetic analog of myelin basic protein (MBP) approved for the treatment of multiple sclerosis, produces a bystander effect on resident astrocytes and microglia by increasing their expression of anti-inflammatory cytokines such as TGF $\beta$ [162]. Alternatively, it has been suggested that $\mathrm{T}$ cells may mediate an indirect effect on adult hippocampal neurogenesis by increasing the production of brain-derived neurotrophic factor (BDNF) [157], which is involved in the proneurogenic actions of $\mathrm{EE}$ [163]. Whether BDNF can counteract the detrimental effects of $\mathrm{T}$ cell depletion on neurogenesis remains unknown. Overall, the roles of microglia in $\mathrm{EE}$ and running-induced neurogenesis are unclear and have to be addressed with more precise experimental designs. In summary, surveillant microglia are part of the physical niche surrounding the neural stem cells and newborn neurons of the mature hippocampus, where they continuously phagocytose the excess of newborn cells. Microglia were also linked to the proneurogenic and anti-inflammatory effects of voluntary running and EE, but direct evidence is missing. The overall contribution of microglia to neurogenesis and learning and memory in normal physiological conditions remains largely unexplored at this early stage in the field.

\section{Conclusion}

In light of these observations, microglia are now emerging as important effector cells during normal brain development and functions, including adult hippocampal neurogenesis. Microglia can exert a positive or negative influence on the proliferation, survival, or differentiation of newborn cells, depending on the inflammatory context. For instance, microglia can compromise the neurogenic cascade during chronic stress, aging, and neurodegenerative diseases, by their release of proinflammatory cytokines such as IL- $1 \beta$, IL-6, and TNF $\alpha$. A reduced fractalkine signalling between neurons and microglia could also be involved during normal aging. However, microglia are not necessarily the only cell type implicated because astrocytes, endothelial cells, mast cells, perivascular and meningeal macrophages, and to a lesser extent neurons and invading peripheral immune cells could further contribute by releasing proinflammatory mediators.

Additionally, microglia were shown to phagocytose the excess of newborn neurons undergoing apoptosis in the hippocampal neurogenic niche during normal physiological conditions, while a similar role in the synaptic integration of newborn cells was also proposed in light of their capacity to phagocytose synaptic elements. Lastly, microglial interactions with $\mathrm{T}$ cells, leading to the release of anti-inflammatory cytokines, neurotrophic factors, and other proneurogenic mediators (notably during EE and voluntary running), could counteract the detrimental effects of inflammation on adult hippocampal neurogenesis and their functional implications for learning and memory.

However, further research is necessary to assess the relative contribution of microglia versus other types of resident and infiltrating inflammatory cells and to determine the nature of the effector cytokines and other inflammatory mediators involved, as well as their cellular and molecular targets in the neurogenic cascade. Such research will undoubtedly help to develop novel strategies aiming at protecting the neurogenic potential and ultimately its essential contribution to learning and memory.

\section{Abbreviations}

\begin{tabular}{|c|c|}
\hline AD: & Alzheimer's disease \\
\hline ANPs: & Amplifying neuroprogenitors \\
\hline APP: & Amyloid precursor protein \\
\hline $\mathrm{A} \beta:$ & Amyloid beta \\
\hline BDNF: & Brain-derived neurotrophic factor \\
\hline BrdU: & 5-Bromo-2'-Deoxyuridine \\
\hline CX3CL1: & Fractalkine \\
\hline CX3CR1: & Fractalkine receptor \\
\hline EAE: & Experimental acute encephalomyelitis \\
\hline EE: & Enriched environment \\
\hline EGF: & Epidermal growth factor \\
\hline FGFb: & Basic fibroblast growth factor \\
\hline GDNF: & Glial cell line-derived neurotrophic factor \\
\hline GFAP: & Glial fibrillary acidic protein \\
\hline GR: & Glucocorticoid receptor \\
\hline HPA: & Hypothalamic-pituitary-adrenal axis \\
\hline ICE: & Interleukin 1 converting enzyme \\
\hline IL-1 $\beta$ : & Interleukin 1 beta \\
\hline IL-1R1: & Interleukin 1 beta receptor \\
\hline IL-4: & Interleukin 4 \\
\hline IL-6: & Interleukin 6 \\
\hline IL-7: & Interleukin 7 \\
\hline IL-11: & Interleukin 11 \\
\hline IFN $\gamma:$ & Interferon gamma \\
\hline IGF-1: & Insulin-like growth factor 1 \\
\hline iNOS: & Inducible nitric oxide synthase \\
\hline LPS: & Bacterial lipopolysaccharides \\
\hline LTP: & Long term potentiation \\
\hline M-CSF: & Macrophage colony-stimulating factor \\
\hline MBP: & Myelin basic protein \\
\hline MHC-II: & Major histocompatibility complex class II \\
\hline MOG: & Myelin oligodendrocyte glycoprotein \\
\hline $\mathrm{NF}-\kappa \mathrm{B}:$ & Nuclear factor \\
\hline & $\begin{array}{l}\text { kappa-light-chain-enhancer of activated B } \\
\text { cells }\end{array}$ \\
\hline NGF: & Nerve growth factor \\
\hline NO: & Nitric oxide \\
\hline NSAID: & Nonsteroidal anti-inflammatory drug \\
\hline NT-4: & Neurotrophin-4 \\
\hline PDGF: & Platelet-derived growth factor \\
\hline PS: & Phosphatidylserine \\
\hline PS1: & Presenilin 1 \\
\hline ROS: & Radical oxygen species \\
\hline
\end{tabular}


SCID: Severe combined immunodeficiency

SGZ: Subgranular zone

TGF $\beta$ : Transforming growth factor beta

TNF $\alpha$ : Tumor necrosis factor alpha

VEGF: Vascular endothelial growth factor.

\section{Conflict of Interests}

The authors declare that there is no conflict of interests regarding the publication of this paper.

\section{Acknowledgments}

This work was supported by grants from the Spanish Ministry of Economy and Competitiveness to Amanda Sierra (BFU2012-32089) and Juan M. Encinas (SAF2012-40085), from Basque Government (Saiotek S-PC 12UN014) and Ikerbasque start-up funds to Juan M. Encinas and Amanda Sierra, and from The Banting Research Foundation, the Scottish Rite Charitable Foundation of Canada, and start-up funds from Université Laval and Centre de recherche du CHU de Québec to Marie-Ève Tremblay.

\section{References}

[1] P. Rezaie and D. Male, "Mesoglia and microglia—a historical review of the concept of mononuclear phagocytes within the central nervous system," Journal of the History of the Neurosciences, vol. 11, no. 4, pp. 325-374, 2002.

[2] F. Ginhoux, S. Lim, G. Hoeffel, D. Low, and T. Huber, "Origin and differentiation of microglia," Frontiers in Cellular Neuroscience, vol. 7, article 45, 2013.

[3] E. Gomez Perdiguero, C. Schulz, and F. Geissmann, "Development and homeostasis of "resident" myeloid cells: the case of the microglia," GLIA, vol. 61, no. 1, pp. 112-120, 2013.

[4] H. Kettenmann, F. Kirchhoff, and A. Verkhratsky, "Microglia: new roles for the synaptic stripper," Neuron, vol. 77, no. 1, pp. 10-18, 2013.

[5] A. Aguzzi, B. A. Barres, and M. L. Bennett, "Microglia: scapegoat, saboteur, or something else?” Science, vol. 339, no. 6116, pp. 156-161, 2013.

[6] K. Helmut, U. K. Hanisch, M. Noda, and A. Verkhratsky, "Physiology of microglia," Physiological Reviews, vol. 91, no. 2, pp. 461-553, 2011.

[7] M. È. Tremblay, B. Stevens, A. Sierra, H. Wake, A. Bessis, and A. Nimmerjahn, "The role of microglia in the healthy brain," Journal of Neuroscience, vol. 31, no. 45, pp. 16064-16069, 2011.

[8] A. Miyamoto, H. Wake, A. J. Moorhouse, and J. Nabekura, "Microglia and synapse interactions: fine tuaning neural circuits and candidate molecules," Frontiers in Cellular Neuroscience, vol. 7, article 70, 2013.

[9] C. Bechade, Y. Cantaut-Belarif, and A. Bessis, "Microglial control of neuronal activity," Frontiers in Cellular Neuroscience, vol. 7, article 32, 2013.

[10] A. Sierra, O. Abiega, A. Shahraz, and H. Neumann, "Janusfaced microglia: beneficial and detrimental consequences of microglial phagocytosis," Frontiers in Cellular Neuroscience, vol. 7, article 6, 2013.
[11] G. Kempermann, S. Jessberger, B. Steiner, and G. Kronenberg, "Milestones of neuronal development in the adult hippocampus," Trends in Neurosciences, vol. 27, no. 8, pp. 447-452, 2004.

[12] J. M. Encinas, T. V. Michurina, N. Peunova et al., "Divisioncoupled astrocytic differentiation and age-related depletion of neural stem cells in the adult hippocampus," Cell Stem Cell, vol. 8, no. 5, pp. 566-579, 2011.

[13] M. A. Bonaguidi, M. A. Wheeler, J. S. Shapiro et al., "In vivo clonal analysis reveals self-renewing and multipotent adult neural stem cell characteristics," Cell, vol. 145, no. 7, pp. 11421155, 2011.

[14] J. J. Breunig, J. I. Arellano, J. D. Macklis, and P. Rakic, "Everything that glitters isn't gold: a critical review of postnatal neural precursor analyses," Cell Stem Cell, vol. 1, no. 6, pp. 612627, 2007.

[15] L. S. Overstreet-Wadiche and G. L. Westbrook, "Functional maturation of adult-generated granule cells," Hippocampus, vol. 16, no. 3, pp. 208-215, 2006.

[16] S. Ge, C. H. Yang, K. S. Hsu, G. L. Ming, and H. Song, "A critical period for enhanced synaptic plasticity in newly generated neurons of the adult brain," Neuron, vol. 54, no. 4, pp. 559-566, 2007.

[17] E. Bruel-Jungerman, C. Rampon, and S. Laroche, "Adult hippocampal neurogenesis, synaptic plasticity and memory: facts and hypotheses," Reviews in the Neurosciences, vol. 18, no. 2, pp. 93-114, 2007.

[18] K. Nakajima, S. Honda, Y. Tohyama, Y. Imai, S. Kohsaka, and T. Kurihara, "Neurotrophin secretion from cultured microglia," Journal of Neuroscience Research, vol. 65, no. 4, pp. 322-331, 2001.

[19] M. Krampert, S. R. Chirasani, F. P. Wachs et al., "Smad7 regulates the adult neural stem/progenitor cell pool in a transforming growth factor $\beta$ - and bone morphogenetic proteinindependent manner," Molecular and Cellular Biology, vol. 30, no. 14, pp. 3685-3694, 2010.

[20] K. I. Mosher, R. H. Andres, T. Fukuhara et al., "Neural progenitor cells regulate microglia functions and activity," Nature Neuroscience, vol. 15, no. 11, pp. 1485-1487, 2012.

[21] V. Tropepe, M. Sibilia, B. G. Ciruna, J. Rossant, E. F. Wagner, and D. van der Kooy, "Distinct neural stem cells proliferate in response to EGF and FGF in the developing mouse telencephalon," Developmental Biology, vol. 208, no. 1, pp. 166-188, 1999.

[22] S. U. Kim and J. De Vellis, "Microglia in health and disease," Journal of Neuroscience Research, vol. 81, no. 3, pp. 302-313, 2005.

[23] B. A. Reynolds and R. L. Rietze, "Neural stem cells and neurospheres-re-evaluating the relationship," Nature Methods, vol. 2, no. 5, pp. 333-336, 2005.

[24] K. Satake, Y. Matsuyama, M. Kamiya et al., "Up-regulation of glial cell line-derived neurotrophic factor (GDNF) following traumatic spinal cord injury," NeuroReport, vol. 11, no. 17, pp. 3877-3881, 2000.

[25] Y. M. Yoo, C. J. Lee, and Y. J. Kim, “Exogenous GDNF increases the migration of the neural stem cells with no protection against kainic acid-induced excitotoxic cell death in rats," Brain Research, vol. 1486, pp. 27-38, 2012.

[26] J. L. Trejo, E. Carro, and I. Torres-Alemán, "Circulating insulinlike growth factor I mediates exercise-induced increases in the number of new neurons in the adult hippocampus," Journal of Neuroscience, vol. 21, no. 5, pp. 1628-1634, 2001. 
[27] K. Striedinger and E. Scemes, "Interleukin- $\beta$ affects calcium signaling and in vitro cell migration of astrocyte progenitors," Journal of Neuroimmunology, vol. 196, no. 1-2, pp. 116-123, 2008.

[28] Y. B. Lee, A. Nagai, and S. U. Kim, "Cytokines, chemokines, and cytokine receptors in human microglia," Journal of Neuroscience Research, vol. 69, no. 1, pp. 94-103, 2002.

[29] G. Kempermann and H. Neumann, "Microglia: the enemy within?” Science, vol. 302, no. 5651, pp. 1689-1690, 2003.

[30] M. F. Mehler, R. Rozental, M. Dougherty, D. C. Spray, and J. A. Kessler, "Cytokine regulation of neuronal differentiation of hippocampal progenitor cells," Nature, vol. 362, no. 6415, pp. 62-65, 1993.

[31] U. Gurok, C. Steinhoff, B. Lipkowitz, H. H. Ropers, C. Scharff, and U. A. Nuber, "Gene expression changes in the course of neural progenitor cell differentiation," Journal of Neuroscience, vol. 24, no. 26, pp. 5982-6002, 2004.

[32] J. B. Demoulin, M. Enarsson, J. Larsson, A. Essaghir, C. H. Heldin, and K. Forsberg-Nilsson, "The gene expression profile of PDGF-treated neural stem cells corresponds to partially differentiated neurons and glia," Growth Factors, vol. 24, no. 3, pp. 184-196, 2006.

[33] F. C. Mansergh, M. A. Wride, and D. E. Rancourt, "Neurons from stem cells: implications for understanding nervous system development and repair," Biochemistry and Cell Biology, vol. 78, no. 5, pp. 613-628, 2000.

[34] F. C. Alcantara Gomes, V. De Oliveira Sousa, and L. Romão, "Emerging roles for TGF- $\beta 1$ in nervous system development," International Journal of Developmental Neuroscience, vol. 23, no. 5, pp. 413-424, 2005.

[35] S. Elkabes, E. M. DiCicco-Bloom, and I. B. Black, "Brain microglia/macrophages express neurotrophins that selectively regulate microglial proliferation and function," Journal of Neuroscience, vol. 16, no. 8, pp. 2508-2521, 1996.

[36] U. K. Hanisch and H. Kettenmann, "Microglia: active sensor and versatile effector cells in the normal and pathologic brain," Nature Neuroscience, vol. 10, no. 11, pp. 1387-1394, 2007.

[37] H. J. Klassen, T. F. Ng, Y. Kurimoto et al., "Multipotent retinal progenitors express developmental markers, differentiate into retinal neurons, and preserve light-mediated behavior," Investigative Ophthalmology and Visual Science, vol. 45, no. 11, pp. 4167-4173, 2004.

[38] W. J. Streit, S. D. Hurley, T. S. McGraw, and S. L. SempleRowland, "Comparative evaluation of cytokine profiles and reactive gliosis supports a critical role for interleukin-6 in neuron-glia signaling during regeneration," Journal of Neuroscience Research, vol. 61, no. 1, pp. 10-20, 2000.

[39] A. Suzumura, M. Sawada, H. Yamamoto, and T. Marunouchi, "Effects of colony stimulating factors on isolated microglia in vitro," Journal of Neuroimmunology, vol. 30, no. 2-3, pp. 111-120, 1990.

[40] Z. Lu, M. R. Elliott, Y. Chen et al., "Phagocytic activity of neuronal progenitors regulates adult neurogenesis," Nature Cell Biology, vol. 13, no. 9, pp. 1076-1084, 2011.

[41] K. Nakajima, Y. Kikuchi, E. Ikoma et al., "Neurotrophins regulate the function of cultured microglia," GLIA, vol. 24, no. 3, pp. 272-289, 1998.

[42] P. A. Lodge and S. Sriram, "Regulation of microglial activation by TGF- $\beta$, IL-10, and CSF-1," Journal of Leukocyte Biology, vol. 60, no. 4, pp. 502-508, 1996.

[43] W. S. Sheng, S. Hu, F. H. Kravitz, P. K. Peterson, and C. C. Chao, "Tumor necrosis factor alpha upregulates human microglial cell production of interleukin-10 in vitro," Clinical and Diagnostic Laboratory Immunology, vol. 2, no. 5, pp. 604-608, 1995.

[44] G. L. Ming and H. Song, "Adult neurogenesis in the mammalian brain: significant answers and significant questions," Neuron, vol. 70, no. 4, pp. 687-702, 2011.

[45] E. Bruel-Jungerman, S. Davis, C. Rampon, and S. Laroche, "Long-term potentiation enhances neurogenesis in the adult dentate gyrus," Journal of Neuroscience, vol. 26, no. 22, pp. 58885893, 2006.

[46] E. Drapeau, M. F. Montaron, S. Aguerre, and D. N. Abrous, "Learning-induced survival of new neurons depends on the cognitive status of aged rats," Journal of Neuroscience, vol. 27, no. 22, pp. 6037-6044, 2007.

[47] A. Mouret, G. Gheusi, M. M. Gabellec, F. De Chaumont, J. C. Olivo-Marin, and P. M. Lledo, "Learning and survival of newly generated neurons: when time matters," Journal of Neuroscience, vol. 28, no. 45, pp. 11511-11516, 2008.

[48] N. Kee, C. M. Teixeira, A. H. Wang, and P. W. Frankland, "Preferential incorporation of adult-generated granule cells into spatial memory networks in the dentate gyrus," Nature Neuroscience, vol. 10, no. 3, pp. 355-362, 2007.

[49] F. Massa, M. Koelh, T. Wiesner et al., "Conditional reduction of adult neurogenesis impairs bidirectional hippocampal synaptic plasticity", Proceedings of the National Academy of Sciences of the United States of America, vol. 108, no. 16, pp. 6644-6649, 2011.

[50] T. J. Shors, G. Miesegaes, A. Beylin, M. Zhao, T. Rydel, and E. Gould, "Neurogenesis in the adult is involved in the formation of trace memories," Nature, vol. 410, no. 6826, pp. 372-376, 2001.

[51] W. Deng, M. D. Saxe, I. S. Gallina, and F. H. Gage, "Adultborn hippocampal dentate granule cells undergoing maturation modulate learning and memory in the brain," Journal of Neuroscience, vol. 29, no. 43, pp. 13532-13542, 2009.

[52] M. Arruda-Carvalho, M. Sakaguchi, K. G. Akers, S. A. Josselyn, and P. W. Frankland, "Posttraining ablation of adult-generated neurons degrades previously acquired memories," Journal of Neuroscience, vol. 31, no. 42, pp. 15113-15127, 2011.

[53] A. Sahay, K. N. Scobie, A. S. Hill et al., "Increasing adult hippocampal neurogenesis is sufficient to improve pattern separation," Nature, vol. 472, no. 7344, pp. 466-470, 2011.

[54] G. L. Ming and H. Song, "Adult neurogenesis in the mammalian central nervous system," Annual Review of Neuroscience, vol. 28, pp. 223-250, 2005.

[55] K. Newton and V. M. Dixit, "Signaling in innate immunity and inflammation," Cold Spring Harbor Perspectives in Biology, vol. 4, no. 3, 2012.

[56] H. K. Jeong, K. Ji, K. Min, and E. H. Joe, "Brain inflammation and microglia: facts and misconceptions," Experimental Neurobiology, vol. 22, no. 2, pp. 59-67, 2013.

[57] D. Stellwagen and R. C. Malenka, "Synaptic scaling mediated by glial TNF- $\alpha$," Nature, vol. 440, no. 7087, pp. 1054-1059, 2006.

[58] M. Pickering, D. Cumiskey, and J. J. O'Connor, "Actions of TNF$\alpha$ on glutamatergic synaptic transmission in the central nervous system," Experimental Physiology, vol. 90, no. 5, pp. 663-670, 2005.

[59] K. Belarbi, T. Jopson, D. Tweedie et al., “TNF- $\alpha$ protein synthesis inhibitor restores neuronal function and reverses cognitive deficits induced by chronic neuroinflammation," Journal of Neuroinflammation, vol. 9, article 23, 2012.

[60] C. T. Ekdahl, J. H. Claasen, S. Bonde, Z. Kokaia, and O. Lindvall, "Inflammation is detrimental for neurogenesis in adult brain," Proceedings of the National Academy of Sciences of the United States of America, vol. 100, no. 23, pp. 13632-13637, 2003. 
[61] M. L. Monje, H. Toda, and T. D. Palmer, "Inflammatory blockade restores adult hippocampal neurogenesis," Science, vol. 302, no. 5651, pp. 1760-1765, 2003.

[62] A. Sierra, J. M. Encinas, J. J. P. Deudero et al., "Microglia shape adult hippocampal neurogenesis through apoptosis-coupled phagocytosis," Cell Stem Cell, vol. 7, no. 4, pp. 483-495, 2010.

[63] D. Chugh, P. Nilsson, S. A. Afjei, A. Bakochi, and C. T. Ekdahl, "Brain inflammation induces post-synaptic changes during early synapse formation in adult-born hippocampal neurons," Experimental Neurobiology, vol. 250, pp. 176-188, 2013.

[64] K. Belarbi, C. Arellano, R. Ferguson, T. Jopson, and S. Rosi, "Chronic neuroinflammation impacts the recruitment of adultborn neurons into behaviorally relevant hippocampal networks," Brain, Behavior, and Immunity, vol. 26, no. 1, pp. 18-23, 2012.

[65] S. Johann, E. Kampmann, B. Denecke et al., "Expression of enzymes involved in the prostanoid metabolism by cortical astrocytes after LPS-induced inflammation," Journal of Molecular Neuroscience, vol. 34, no. 2, pp. 177-185, 2008.

[66] P. V. B. Reddy, K. V. Rama Rao, and M. D. Norenberg, "Inhibitors of the mitochondrial permeability transition reduce ammonia-induced cell swelling in cultured astrocytes," Journal of Neuroscience Research, vol. 87, no. 12, pp. 2677-2685, 2009.

[67] H. Nie, H. Zhang, and H. R. Weng, "Minocycline prevents impaired glial glutamate uptake in the spinal sensory synapses of neuropathic rats," Neuroscience, vol. 170, no. 3, pp. 901-912, 2010.

[68] F. Cerbai, D. Lana, D. Nosi et al., "The neuron-astrocytemicroglia triad in normal brain ageing and in a model of neuroinflammation in the rat hippocampus," PLoS ONE, vol. 7, no. 9, Article ID e45250, 2012.

[69] W. J. Jin, S. W. Feng, Z. Feng, S. M. Lu, T. Qi, and Y. N. Qian, "Minocycline improves postoperative cognitive impairment in aged mice by inhibiting astrocytic activation," NeuroReport, 2013.

[70] K. A. Ji, M. S. Yang, H. K. Jeong et al., "Resident microglia die and infiltrated neutrophils and monocytes become major inflammatory cells in lipopolysaccharide-injected brain," GLIA, vol. 55, no. 15, pp. 1577-1588, 2007.

[71] N. J. van Wagoner, J. W. Oh, P. Repovic, and E. N. Benveniste, "Interleukin-6 (IL-6) production by astrocytes: autocrine regulation by IL-6 and the soluble IL-6 receptor," Journal of Neuroscience, vol. 19, no. 13, pp. 5236-5244, 1999.

[72] L. Valliéres, I. L. Campbell, F. H. Gage, and P. E. Sawchenko, "Reduced hippocampal neurogenesis in adult transgenic mice with chronic astrocytic production of interleukin-6," Journal of Neuroscience, vol. 22, no. 2, pp. 486-492, 2002.

[73] B. S. McEwen, "Physiology and neurobiology of stress and adaptation: central role of the brain," Physiological Reviews, vol. 87, no. 3, pp. 873-904, 2007.

[74] V. Luine, M. Villegas, C. Martinez, and B. S. McEwen, "Repeated stress causes reversible impairments of spatial memory performance," Brain Research, vol. 639, no. 1, pp. 167-170, 1994.

[75] S. Rivest, "Molecular insights on the cerebral innate immune system," Brain, Behavior, and Immunity, vol. 17, no. 1, pp. 13-19, 2003.

[76] S. Nadeau and S. Rivest, "Endotoxemia prevents the cerebral inflammatory wave induced by intraparenchymal lipopolysaccharide injection: role of glucocorticoids and CD14," Journal of Immunology, vol. 169, no. 6, pp. 3370-3381, 2002.
[77] A. Sierra, A. Gottfried-Blackmore, T. A. Milner, B. S. McEwen, and K. Bulloch, "Steroid hormone receptor expression and function in microglia," GLIA, vol. 56, no. 6, pp. 659-674, 2008.

[78] C. Liston and W. B. Gan, "Glucocorticoids are critical regulators of dendritic spine development and plasticity in vivo," Proceedings of the National Academy of Sciences of the United States of America, vol. 108, no. 38, pp. 16074-16079, 2011.

[79] C. Liston, J. M. Cichon, F. Jeanneteau, Z. Jia, M. V. Chao, and W. B. Gan, "Circadian glucocorticoid oscillations promote learning-dependent synapse formation and maintenance," Nature Neuroscience, vol. 16, no. 6, pp. 698-705, 2013.

[80] M. A. Carrillo-de Sauvage, L. Maatouk, I. Arnoux et al., "Potent and multiple regulatory actions of microglial glucocorticoid receptors during CNS inflammation," Cell Death \& Differentiation, vol. 20, no. 11, pp. 1546-1557, 2013.

[81] S. F. Sorrells, J. R. Caso, C. D. Munhoz, and R. M. Sapolsky, “The stressed CNS: when glucocorticoids aggravate inflammation," Neuron, vol. 64, no. 1, pp. 33-39, 2009.

[82] M. A. Bellavance and S. Rivest, "The neuroendocrine control of the innate immune system in health and brain diseases," Immunological Reviews, vol. 248, no. 1, pp. 36-55, 2012.

[83] R. J. Tynan, S. Naicker, M. Hinwood et al., "Chronic stress alters the density and morphology of microglia in a subset of stressresponsive brain regions," Brain, Behavior, and Immunity, vol. 24, no. 7, pp. 1058-1068, 2010.

[84] E. S. Wohleb, N. D. Powell, J. P. Godbout, and J. F. Sheridan, "Stress-induced recruitment of bone marrow-derived monocytes to the brain promotes anxiety-like behavior," The Journal of Neuroscience, vol. 33, no. 34, pp. 13820-13833, 2013.

[85] Y. Diz-Chaves, M. Astiz, M. J. Bellini, and L. M. Garcia-Segura, "Prenatal stress increases the expression of proinflammatory cytokines and exacerbates the inflammatory response to LPS in the hippocampal formation of adult male mice," Brain, Behavior, and Immunity, vol. 28, pp. 196-206, 2013.

[86] E. S. Wohleb, A. M. Fenn, A. M. Pacenta, N. D. Powell, J. F. Sheridan, and J. P. Godbout, "Peripheral innate immune challenge exaggerated microglia activation, increased the number of inflammatory CNS macrophages, and prolonged social withdrawal in socially defeated mice," Psychoneuroendocrinology, vol. 37, no. 9, pp. 1491-1505, 2012.

[87] C. Mirescu and E. Gould, "Stress and adult neurogenesis," Hippocampus, vol. 16, no. 3, pp. 233-238, 2006.

[88] J. L. Warner-Schmidt and R. S. Duman, "Hippocampal neurogenesis: opposing effects of stress and antidepressant treatment," Hippocampus, vol. 16, no. 3, pp. 239-249, 2006.

[89] N. D. Hanson, M. J. Owens, K. A. Boss-Williams, J. M. Weiss, and C. B. Nemeroff, "Several stressors fail to reduce adult hippocampal neurogenesis," Psychoneuroendocrinology, vol. 36, no. 10, pp. 1520-1529, 2011.

[90] E. Gould, B. S. McEwen, P. Tanapat, L. A. M. Galea, and E. Fuchs, "Neurogenesis in the dentate gyrus of the adult tree shrew is regulated by psychosocial stress and NMDA receptor activation," Journal of Neuroscience, vol. 17, no. 7, pp. 2492-2498, 1997.

[91] J. W. Koo and R. S. Duman, "IL-1 $\beta$ is an essential mediator of the antineurogenic and anhedonic effects of stress," Proceedings of the National Academy of Sciences of the United States of America, vol. 105, no. 2, pp. 751-756, 2008.

[92] D. C. Lagace, M. H. Donovan, N. A. Decarolis et al., "Adult hippocampal neurogenesis is functionally important for stressinduced social avoidance," Proceedings of the National Academy 
of Sciences of the United States of America, vol. 107, no. 9, pp. 4436-4441, 2010.

[93] G. Kronenberg, I. Kirste, D. Inta et al., "Reduced hippocampal neurogenesis in the GR+/- genetic mouse model of depression," European Archives of Psychiatry and Clinical Neuroscience, vol. 259, no. 8, pp. 499-504, 2009.

[94] M. B. Howren, D. M. Lamkin, and J. Suls, "Associations of depression with c-reactive protein, IL-1, and IL-6: a metaanalysis," Psychosomatic Medicine, vol. 71, no. 2, pp. 171-186, 2009.

[95] J. W. Koo, S. J. Russo, D. Ferguson, E. J. Nestler, and R. S. Duman, "Nuclear factor $-\kappa \mathrm{B}$ is a critical mediator of stressimpaired neurogenesis and depressive behavior," Proceedings of the National Academy of Sciences of the United States of America, vol. 107, no. 6, pp. 2669-2674, 2010.

[96] I. Goshen, T. Kreisel, O. Ben-Menachem-Zidon et al., "Brain interleukin-1 mediates chronic stress-induced depression in mice via adrenocortical activation and hippocampal neurogenesis suppression," Molecular Psychiatry, vol. 13, no. 7, pp. 717$728,2008$.

[97] C. D. Munhoz, L. B. Lepsch, E. M. Kawamoto et al., "Chronic unpredictable stress exacerbates lipopolysaccharide-induced activation of nuclear factor- $\kappa \mathrm{B}$ in the frontal cortex and hippocampus via glucocorticoid secretion," Journal of Neuroscience, vol. 26, no. 14, pp. 3813-3820, 2006.

[98] S. J. Sukoff Rizzo, S. J. Neal, Z. A. Hughes et al., "Evidence for sustained elevation of IL- 6 in the CNS as a key contributor of depressive-like phenotypes," Translational Psychiatry, vol. 2, article e199, 2012.

[99] A. Garcia, B. Steiner, G. Kronenberg, A. Bick-Sander, and G. Kempermann, "Age-dependent expression of glucocorticoidand mineralocorticoid receptors on neural precursor cell populations in the adult murine hippocampus," Aging Cell, vol. 3, no. 6, pp. 363-371, 2004.

[100] M. G. Frank, B. M. Thompson, L. R. Watkins, and S. F. Maier, "Glucocorticoids mediate stress-induced priming of microglial pro-inflammatory responses," Brain, Behavior, and Immunity, vol. 26, no. 2, pp. 337-345, 2012.

[101] S. Sugama, M. Fujita, M. Hashimoto, and B. Conti, "Stress induced morphological microglial activation in the rodent brain: involvement of interleukin-18," Neuroscience, vol. 146, no. 3, pp. 1388-1399, 2007.

[102] A. Sierra, J. M. Encinas, and M. Maletic-Savatic, "Adult human neurogenesis: from microscopy to magnetic resonance imaging," Frontiers in Neuroscience, vol. 5, article 47, 2011.

[103] H. G. Kuhn, H. Dickinson-Anson, and F. H. Gage, "Neurogenesis in the dentate gyrus of the adult rat: age-related decrease of neuronal progenitor proliferation," Journal of Neuroscience, vol. 16, no. 6, pp. 2027-2033, 1996.

[104] L. N. Manganas, X. Zhang, Y. Li et al., "Magnetic resonance spectroscopy identifies neural progenitor cells in the live human brain," Science, vol. 318, no. 5852, pp. 980-985, 2007.

[105] K. L. Spalding, O. Bergmann, K. Alkass et al., "Dynamics of hippocampal neurogenesis in adult humans," Cell, vol. 153, no. 6, pp. 1219-1227, 2013.

[106] J. M. Encinas and A. Sierra, "Neural stem cell deforestation as the main force driving the age-related decline in adult hippocampal neurogenesis," Behavioural Brain Research, vol. 227, no. 2, pp. 433-439, 2012.

[107] K. S. Krabbe, M. Pedersen, and H. Bruunsgaard, "Inflammatory mediators in the elderly," Experimental Gerontology, vol. 39, no. 5, pp. 687-699, 2004.
[108] B. S. Diniz, A. L. Teixeira, L. Talib, W. F. Gattaz, and O. V. Forlenza, "Interleukin-1 $\beta$ serum levels is increased in antidepressant-free elderly depressed patients," American Journal of Geriatric Psychiatry, vol. 18, no. 2, pp. 172-176, 2010.

[109] A. Sierra, A. C. Gottfried-Blackmore, B. S. Mcewen, and K. Bulloch, "Microglia derived from aging mice exhibit an altered inflammatory profile," GLIA, vol. 55, no. 4, pp. 412-424, 2007.

[110] C. Franceschi, M. Bonafè, S. Valensin et al., "Inflamm-aging. An evolutionary perspective on immunosenescence," Annals of the New York Academy of Sciences, vol. 908, pp. 244-254, 2000.

[111] R. Sano and J. C. Reed, "ER stress-induced cell death mechanisms," Biochimica et Biophysica Acta, vol. 1833, no. 12, pp. 34603470, 2013

[112] S. Z. Hasnain, R. Lourie, I. Das, A. C. H. Chen, and M. A. McGuckin, "The interplay between endoplasmic reticulum stress and inflammation," Immunology and Cell Biology, vol. 90, no. 3, pp. 260-270, 2012.

[113] S. Matus, L. H. Glimcher, and C. Hetz, "Protein folding stress in neurodegenerative diseases: a glimpse into the ER," Current Opinion in Cell Biology, vol. 23, no. 2, pp. 239-252, 2011.

[114] Y. Ito, M. Yamada, H. Tanaka et al., "Involvement of CHOP, an ER-stress apoptotic mediator, in both human sporadic ALS and ALS model mice," Neurobiology of Disease, vol. 36, no. 3, pp. 470-476, 2009.

[115] D. Papadimitriou, V. Le Verche, A. Jacquier, B. Ikiz, S. Przedborski, and D. B. Re, "Inflammation in ALS and SMA: sorting out the good from the evil," Neurobiology of Disease, vol. 37, no. 3, pp. 493-502, 2010.

[116] A. R. Johnson, J. J. Milner, and L. Makowski, “The inflammation highway: metabolism accelerates inflammatory traffic in obesity," Immunological Reviews, vol. 249, no. 1, pp. 218-238, 2012.

[117] S. Buch, H. Yao, M. Guo et al., "Cocaine and HIV-1 interplay in CNS: cellular and molecular mechanisms," Current HIV Research, vol. 10, no. 5, pp. 425-428, 2012.

[118] M. K. Brown and N. Naidoo, "The endoplasmic reticulum stress response in aging and age-related diseases," Frontiers in Physiology, vol. 3, article 263, 2012.

[119] G. Castro, C. Areias MF, L. Weissmann et al., "Diet-induced obesity induces endoplasmic reticulum stress and insulin resistance in the amygdala of rats," FEBS Open Bio, vol. 3, pp. 443449, 2013.

[120] A. A. Pavlovsky, D. Boehning, D. Li, Y. Zhang, X. Fan, and T. A. Green, "Psychological stress, cocaine and natural reward each induce endoplasmic reticulum stress genes in rat brain," Neuroscience, vol. 246, pp. 160-169, 2013.

[121] G. Gargiulo, M. Cesaroni, M. Serresi et al., "In vivo RNAi screen for BMI1 targets identifies TGF-beta/BMP-ER stress pathways as key regulators of neural- and malignant glioma-stem cell homeostasis," Cancer Cell, vol. 23, no. 5, pp. 660-676, 2013.

[122] A. Salminen, K. Kaarniranta, and A. Kauppinen, "Inflammaging: disturbed interplay between autophagy and inflammasomes," Aging, vol. 4, no. 3, pp. 166-175, 2012.

[123] E. Latz, T. S. Xiao, and A. Stutz, "Activation and regulation of the inflammasomes," Nature Reviews Immunology, vol. 13, no. 6, pp. 397-411, 2013.

[124] C. Gemma, A. D. Bachstetter, M. J. Cole, M. Fister, C. Hudson, and P. C. Bickford, "Blockade of caspase-1 increases neurogenesis in the aged hippocampus," European Journal of Neuroscience, vol. 26, no. 10, pp. 2795-2803, 2007.

[125] M. D. Wu, A. M. Hein, M. J. Moravan, S. S. Shaftel, J. A. Olschowka, and M. K. O'Banion, "Adult murine hippocampal 
neurogenesis is inhibited by sustained IL- $1 \beta$ and not rescued by voluntary running," Brain, Behavior, and Immunity, vol. 26, no. 2, pp. 292-300, 2012.

[126] Y. Wolf, S. Yona, K. W. Kim, and S. Jung, "Microglia, seen from the CX3CR1 angle," Frontiers in Cellular Neuroscience, vol. 7, article 26, 2013.

[127] A. E. Cardona, E. P. Pioro, M. E. Sasse et al., "Control of microglial neurotoxicity by the fractalkine receptor," Nature Neuroscience, vol. 9, no. 7, pp. 917-924, 2006.

[128] K. Bhaskar, M. Konerth, O. N. Kokiko-Cochran, A. Cardona, R. M. Ransohoff, and B. T. Lamb, "Regulation of tau pathology by the microglial fractalkine receptor," Neuron, vol. 68, no. 1, pp. 19-31, 2010.

[129] A. D. Bachstetter, J. M. Morganti, J. Jernberg et al., "Fractalkine and CX3CR1 regulate hippocampal neurogenesis in adult and aged rats," Neurobiology of Aging, vol. 32, no. 11, pp. 2030-2044, 2011.

[130] S. Jung, J. Aliberti, P. Graemmel et al., "Analysis of fractalkine receptor CX3CR1 function by targeted deletion and green fluorescent protein reporter gene insertion," Molecular and Cellular Biology, vol. 20, no. 11, pp. 4106-4114, 2000.

[131] J. T. Rogers, J. M. Morganti, A. D. Bachstetter et al., "CX3CR1 deficiency leads to impairment of hippocampal cognitive function and synaptic plasticity," Journal of Neuroscience, vol. 31, no. 45, pp. 16241-16250, 2011.

[132] W. S. T. Griffin, L. C. Stanley, C. Ling et al., "Brain interleukin 1 and S-100 immunoreactivity are elevated in Down syndrome and Alzheimer disease," Proceedings of the National Academy of Sciences of the United States of America, vol. 86, no. 19, pp. 7611$7615,1989$.

[133] W. C. Benzing, J. R. Wujek, E. K. Ward et al., "Evidence for glialmediated inflammation in aged $\mathrm{APP}(\mathrm{SW})$ transgenic mice," Neurobiology of Aging, vol. 20, no. 6, pp. 581-589, 1999.

[134] T. J. Seabrook, L. Jiang, M. Maier, and C. A. Lemere, "Minocycline affects microglia activation, $\mathrm{A} \beta$ deposition, and behavior in APP-tg mice," GLIA, vol. 53, no. 7, pp. 776-782, 2006.

[135] B. Biscaro, O. Lindvall, G. Tesco, C. T. Ekdahl, and R. M. Nitsch, "Inhibition of microglial activation protects hippocampal neurogenesis and improves cognitive deficits in a transgenic mouse model for Alzheimer's disease," Neurodegenerative Diseases, vol. 9, no. 4, pp. 187-198, 2012.

[136] P. A. Zunszain, C. Anacker, A. Cattaneo et al., "Interleukin-1 $\beta$ : a new regulator of the kynurenine pathway affecting human hippocampal neurogenesis," Neuropsychopharmacology, vol. 37, no. 4, pp. 939-949, 2012.

[137] R. De Simone, M. Antonietta Ajmone-Cat, P. Tirassa, and L. Minghetti, "Apoptotic PC12 cells exposing phosphatidylserine promote the production of anti-inflammatory and neuroprotective molecules by microglial cells," Journal of Neuropathology and Experimental Neurology, vol. 62, no. 2, pp. 208-216, 2003.

[138] M. S. Buckwalter, M. Yamane, B. S. Coleman et al., "Chronically increased transforming growth factor- $\beta 1$ strongly inhibits hippocampal neurogenesis in aged mice," American Journal of Pathology, vol. 169, no. 1, pp. 154-164, 2006.

[139] O. Butovsky, Y. Ziv, A. Schwartz et al., "Microglia activated by IL- 4 or IFN- $\gamma$ differentially induce neurogenesis and oligodendrogenesis from adult stem/progenitor cells," Molecular and Cellular Neuroscience, vol. 31, no. 1, pp. 149-160, 2006.

[140] C. T. Ekdahl, "Microglial activation-tuning and pruning adult neurogenesis," Frontiers in Pharmacology, vol. 3, article 41, 2012.
[141] H. Wake, A. J. Moorhouse, S. Jinno, S. Kohsaka, and J. Nabekura, "Resting microglia directly monitor the functional state of synapses in vivo and determine the fate of ischemic terminals," Journal of Neuroscience, vol. 29, no. 13, pp. 3974-3980, 2009.

[142] M. È. Tremblay, R. L. Lowery, and A. K. Majewska, "Microglial interactions with synapses are modulated by visual experience," PLoS Biology, vol. 8, no. 11, Article ID e1000527, 2010.

[143] M. È. Tremblay, M. L. Zettel, J. R. Ison, P. D. Allen, and A. K. Majewska, "Effects of aging and sensory loss on glial cells in mouse visual and auditory cortices," GLIA, vol. 60, no. 4, pp. 541-558, 2012.

[144] D. P. Schafer, E. K. Lehrman, A. G. Kautzman et al., "Microglia sculpt postnatal neural circuits in an activity and complementdependent manner," Neuron, vol. 74, no. 4, pp. 691-705, 2012.

[145] H. Nakanishi, "Microglial functions and proteases," Molecular Neurobiology, vol. 27, no. 2, pp. 163-176, 2003.

[146] M. È. Tremblay and A. K. Majewska, "A role for microglia in synaptic plasticity?" Communicative and Integrative Biology, vol. 4, no. 2, pp. 220-222, 2011.

[147] R. C. Paolicelli, G. Bolasco, F. Pagani et al., "Synaptic pruning by microglia is necessary for normal brain development," Science, vol. 333, no. 6048, pp. 1456-1458, 2011.

[148] G. Kempermann, H. G. Kuhn, and F. H. Gage, "More hippocampal neurons in adult mice living in an enriched environment," Nature, vol. 386, no. 6624, pp. 493-495, 1997.

[149] M. Nilsson, E. Perfilieva, U. Johansson, O. Orwar, and P. S. Eriksson, "Enriched environment increases neurogenesis in the adult rat dentate gyrus and improves spatial memory," Journal of Neurobiology, vol. 39, no. 4, pp. 569-578, 1999.

[150] H. van Praag, G. Kempermann, and F. H. Gage, "Running increases cell proliferation and neurogenesis in the adult mouse dentate gyrus," Nature Neuroscience, vol. 2, no. 3, pp. 266-270, 1999.

[151] D. Young, P. A. Lawlor, P. Leone, M. Dragunow, and M. J. During, "Environmental enrichment inhibits spontaneous apoptosis, prevents seizures and is neuroprotective," Nature Medicine, vol. 5, no. 4, pp. 448-453, 1999.

[152] L. L. Williamson, A. Chao, and S. D. Bilbo, "Environmental enrichment alters glial antigen expression and neuroimmune function in the adult rat hippocampus," Brain, Behavior, and Immunity, vol. 26, no. 3, pp. 500-510, 2012.

[153] L. Maggi, M. Scianni, I. Branchi, I. D’Andrea, C. Lauro, and C. Limatola, "CX(3)CR1 deficiency alters hippocampal-dependent plasticity phenomena blunting the effects of enriched environment," Frontiers in Cellular Neuroscience, vol. 5, article 22, 2011.

[154] I. Goshen, A. Avital, T. Kreisel, T. Licht, M. Segal, and R. Yirmiya, "Environmental enrichment restores memory functioning in mice with impaired IL-1 signaling via reinstatement of long-term potentiation and spine size enlargement," Journal of Neuroscience, vol. 29, no. 11, pp. 3395-3403, 2009.

[155] Y. Ziv, N. Ron, O. Butovsky et al., "Immune cells contribute to the maintenance of neurogenesis and spatial learning abilities in adulthood," Nature Neuroscience, vol. 9, no. 2, pp. 268-275, 2006.

[156] J. Kipnis, H. Cohen, M. Cardon, Y. Ziv, and M. Schwartz, “T cell deficiency leads to cognitive dysfunction: implications for therapeutic vaccination for schizophrenia and other psychiatric conditions," Proceedings of the National Academy of Sciences of the United States of America, vol. 101, no. 21, pp. 8180-8185, 2004.

[157] S. A. Wolf, B. Steiner, A. Akpinarli et al., "CD4-positive T lymphocytes provide a neuroimmunological link in the control 
of adult hippocampal neurogenesis," Journal of Immunology, vol. 182, no. 7, pp. 3979-3984, 2009.

[158] G. J. Huang, A. L. Smith, D. H. D. Gray et al., "A genetic and functional relationship between $\mathrm{T}$ cells and cellular proliferation in the adult hippocampus," PLOS Biology, vol. 8, no. 12, Article ID e1000561, 2010.

[159] R. M. Ransohoff and B. Engelhardt, "The anatomical and cellular basis of immune surveillance in the central nervous system," Nature Reviews Immunology, vol. 12, no. 9, pp. 623-635, 2012.

[160] M. Olah, G. Ping, A. H. De Haas et al., "Enhanced hippocampal neurogenesis in the absence of microglia $\mathrm{T}$ cell interaction and microglia activation in the murine running wheel model," GLIA, vol. 57, no. 10, pp. 1046-1061, 2009.

[161] E. Gebara, S. Sultan, J. Kocher-Braissant, and N. Toni, "Adult hippocampal neurogenesis inversely correlates with microglia in conditions of voluntary running and aging," Frontiers in Neuroscience, vol. 7, article 145, 2013.

[162] R. Aharoni, B. Kayhan, R. Eilam, M. Sela, and R. Arnon, "Glatiramer acetate-specific T cells in the brain express Thelper 2/3 cytokines and brain-derived neurotrophic factor in situ," Proceedings of the National Academy of Sciences of the United States of America, vol. 100, no. 2, pp. 14157-14162, 2003.

[163] C. Rossi, A. Angelucci, L. Costantin et al., "Brain-derived neurotrophic factor (BDNF) is required for the enhancement of hippocampal neurogenesis following environmental enrichment," European Journal of Neuroscience, vol. 24, no. 7, pp. 18501856, 2006. 

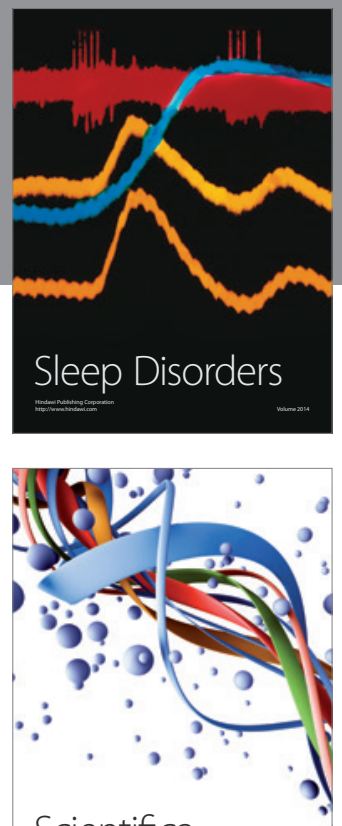

Scientifica
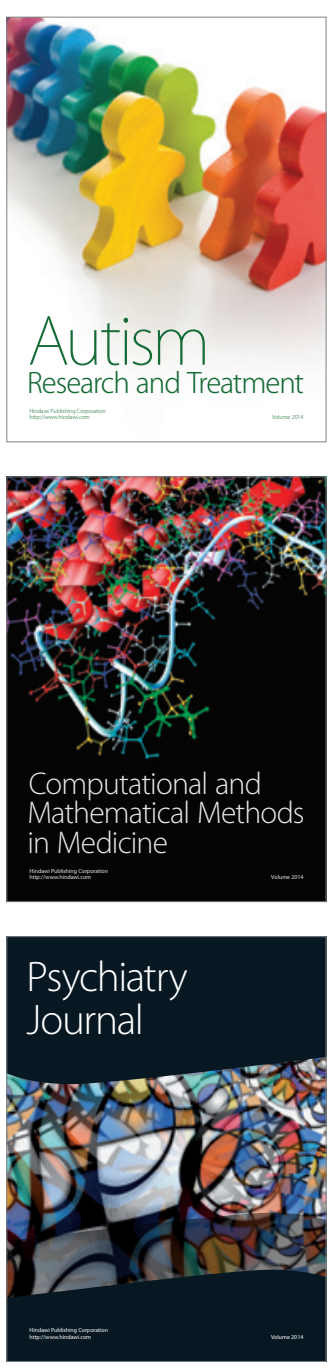
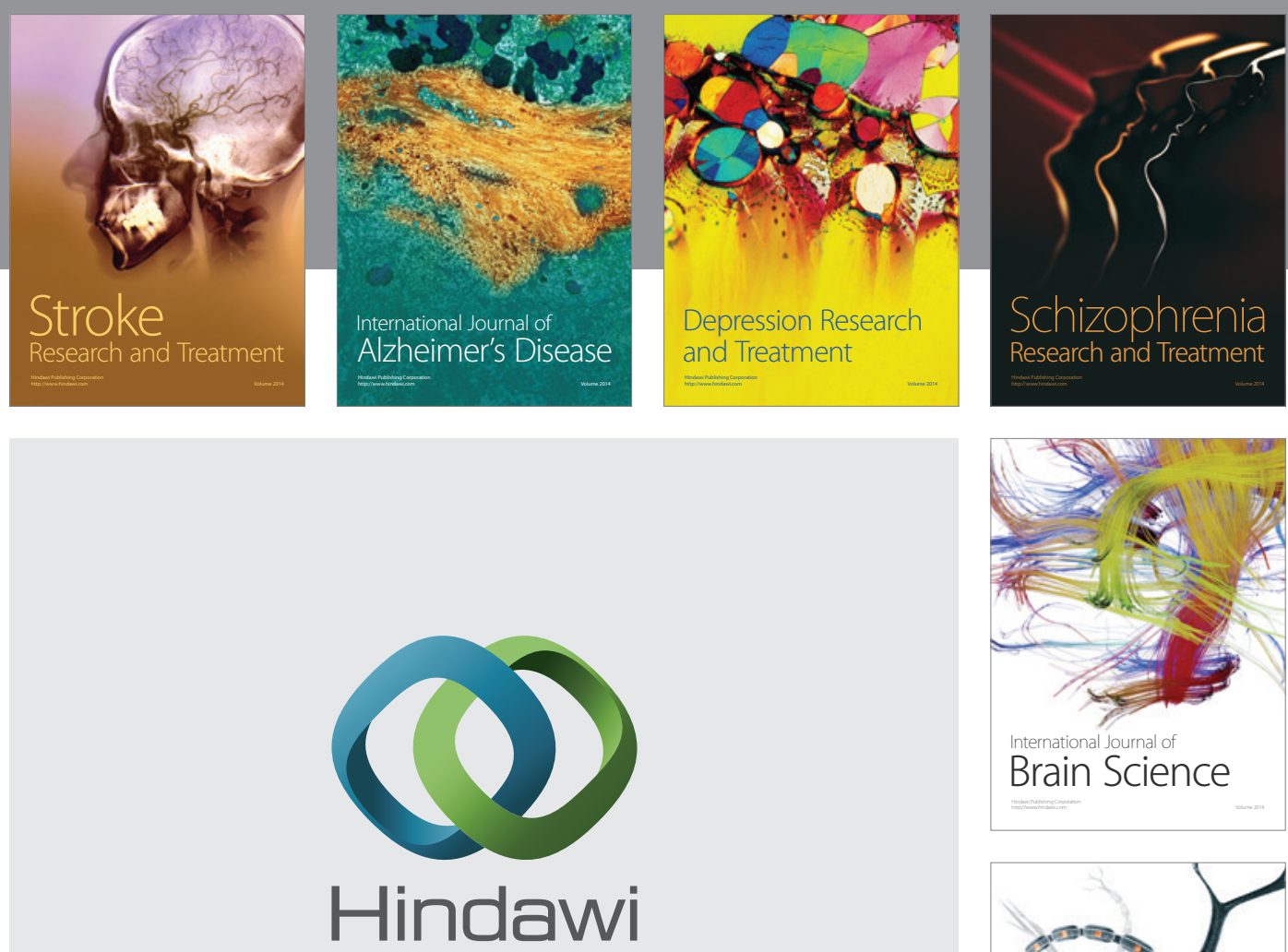

Submit your manuscripts at

http://www.hindawi.com
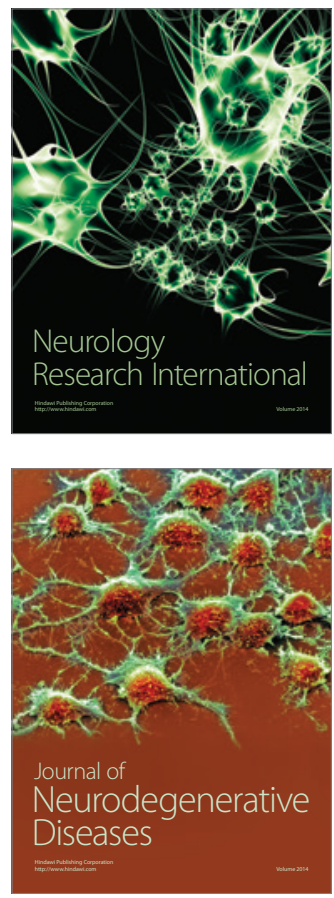

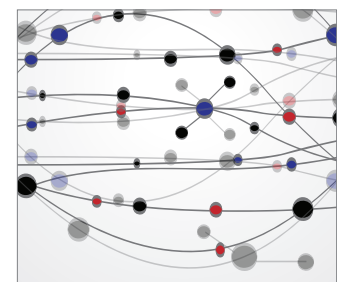

The Scientific World Journal
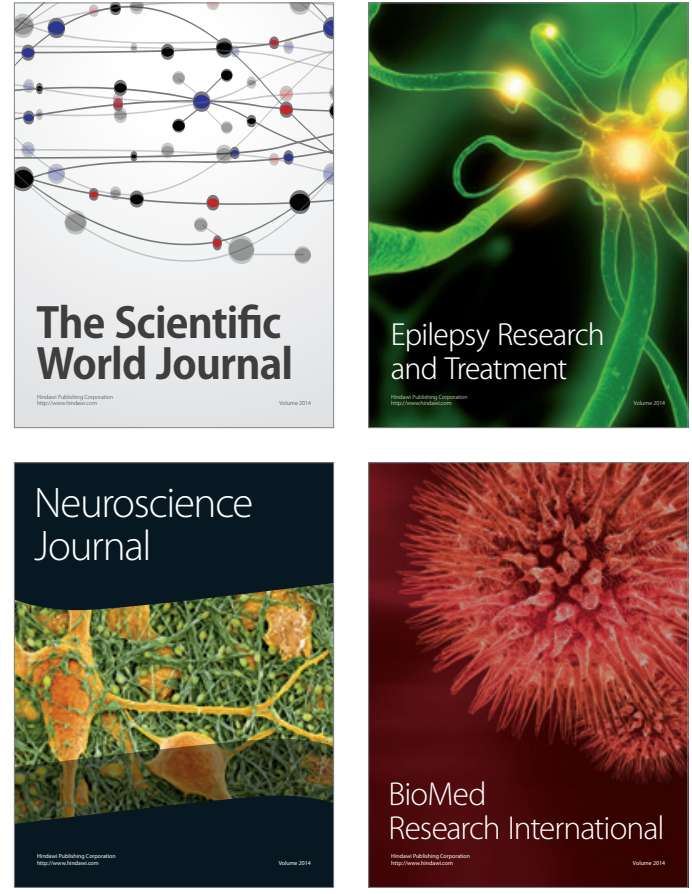

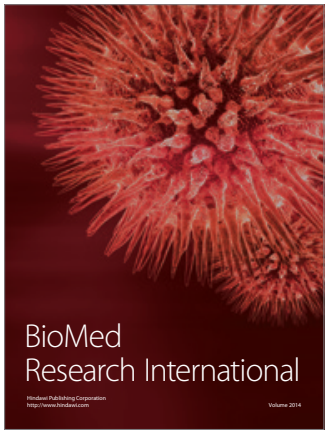

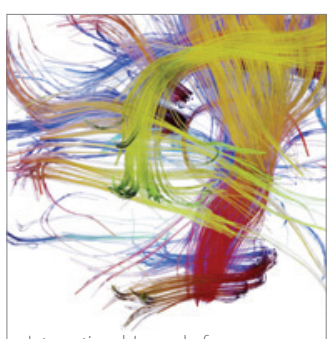

Brain Science

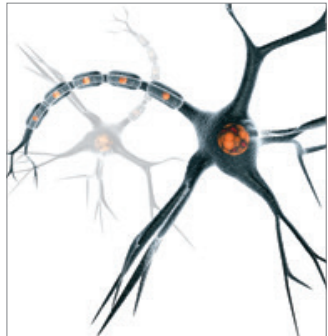

Neural Plasticity
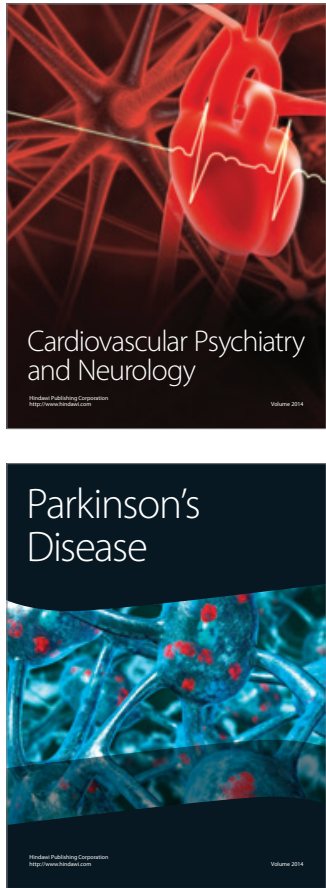